\title{
Drug evaluation based on phosphomimetic PDHA1 reveals the complexity of activity-related cell death in A549 non-small cell lung cancer cells
}

\author{
Ling Jin ${ }^{1,2}$, Minkyoung $\mathrm{Cho}^{2}$, Bo-Sung Kim ${ }^{1,2}$, Jung Ho Han ${ }^{1,2}$, Sungmi Park ${ }^{3}$, In-Kyu Lee ${ }^{3,4}$, Dongryeol Ryu ${ }^{5}$, Jae Ho Kim ${ }^{6}$, \\ Sung-Jin Bae $e^{2, *} \mathcal{E}$ Ki-Tae Ha, ${ }^{1,2, *}$ \\ ${ }^{1}$ Department of Korean Medical Science, School of Korean Medicine, Pusan National University, Yangsan 50612, ${ }^{2}$ Korean Medical Research \\ Center for Healthy Aging, Pusan National University, Yangsan 50612, ${ }^{3}$ Department of Internal Medicine, School of Medicine, Kyungpook \\ National University, Daegu 41944, ${ }^{4}$ Research Institute of Aging and Metabolism, Kyungpook National University, Daegu 41404, \\ ${ }^{5}$ Department of Molecular Cell Biology, School of Medicine, Sungkyunkwan University, Suwon 16419, ${ }^{6}$ Department of Physiology, College \\ of Medicine, Pusan National University, Yangsan 50612, Korea
}

\begin{abstract}
Cancer cells predominantly generate energy via glycolysis, even in the presence of oxygen, to support abnormal cell proliferation. Suppression of PDHA1 by PDK1 prevents the conversion of cytoplasmic pyruvate into Acetyl-CoA. Several PDK inhibitors have been identified, but their clinical applications have not been successful for unclear reasons. In this study, endogenous PDHA1 in A549 cells was silenced by the CRISPR/Cas9 system, and PDHA1 ${ }^{\text {WT }}$ and PDHA1 ${ }^{3 \mathrm{SD}}$ were transduced. Since PDHA1 ${ }^{\text {SSD }}$ cannot be phosphorylated by PDKs, it was used to evaluate the specific activity of PDK inhibitors. This study highlights that PDHA1 ${ }^{\text {WT }}$ and PDHA1 ${ }^{3 \mathrm{SD}}$ A549 cells can be used as a cell-based PDK inhibitor-distinction system to examine the relationship between PDH activity and cell death by established PDK inhibitors. Leelamine, huzhangoside $A$ and otobaphenol induced PDH activity-dependent apoptosis, whereas AZD7545, VER-246608 and DCA effectively enhanced PDHA1 activity but little toxic to cancer cells. Furthermore, the activity of phosphomimetic PDHA1 revealed the complexity of its regulation, which requires further in-depth investigation. [BMB Reports 2021; 54(11): 563-568]
\end{abstract}

\section{INTRODUCTION}

Normal cells rely on mitochondrial oxidative phosphorylation

${ }^{*}$ Corresponding authors. Sung-Jin Bae, Tel: +82-51-510-8434; Fax: +82-51-510-8437; E-mail: Dr.NowOrNever@pusan.ac.kr; Ki-Tae Ha, Tel: +82-51-510-8464; Fax: +82-51-510-8420; E-mail: hagis@pusan. ac.kr

https://doi.org/10.5483/BMBRep.2021.54.11.101

Received 26 July 2021, Revised 17 August 2021, Accepted 30 August 2021

Keywords: Apoptosis, Glycolysis, PDHA1, PDK, Warburg effect
(OXPHOS) to generate adenosine triphosphate (ATP) (1). Conversely, cancer cells mainly generate energy via glycolysis to support the abnormal cell proliferation even in the presence of oxygen $(2,3)$. Upregulation of glycolysis is one of the adaptation processes observed in tumors in response to environmental pressures such as hypoxia and acidosis (4). Therefore, inhibition of glycolysis has been regarded as a promising therapeutic strategy against cancer cells (5).

In glucose metabolism, the pyruvate dehydrogenase (PDH) complex (PDC) mediates a major regulatory step, linking glycolysis to tricyclic acid cycle through catalyzing pyruvate to acetylCoA (6). Previous study suggested that inhibition of PDH activity provides advantages for cancer growth (7). PDH kinases (PDK1-4) regulate $\mathrm{PDH}$ activity by phosphorylating $\mathrm{PDHA} 1$ at three individual serine (Ser, S) residues (S293, S300 and S232) (8). PDK1 is frequently increased in cancer cells and plays an important role in tilting the energy balance in their favor by enhancing the Warburg effect (9). Suppression of PDK1 increases mitochondrial oxygen consumption and induces cancer cell death frequently by activating apoptosis (10). Therefore, PDK1 inhibition could serve as a novel therapeutic approach for treating cancers.

Through experiments or clinical trials, several PDK inhibitors have been developed, such as AZD7545, JX06, VER-246608, dichloroacetate (DCA) and Leelamine (11-13). We have also identified several novel PDK1 inhibitors including huzhangoside $\mathrm{A}$, ilimaquinone and hemistepsin $\mathrm{A}$, isolated from Anemone rivularis, Smenospongia cerebriformis and Hemistepta lyrate, respectively (14-16). These PDK inhibitors enhanced the metabolic shift from glycolysis to OXPHOS and consequently induced mitochondrial ROS-mediated apoptosis in several cancer cells (14-16). To date, there are no effective PDK inhibitors used for cancer treatment in clinical practice. Although extensive studies have been in progress to find novel PDK inhibitors, target accuracy and off-target toxicity of the inhibitors have made them controversial.

ISSN: 1976-670X (electronic edition)

Copyright (C) 2021 by the The Korean Society for Biochemistry and Molecular Biology

(c) This is an open-access article distributed under the terms of the Creative Commons Attribution Non-Commercial License (http://creativecommons.org/licenses/by-nc/4.0) which permits unrestricted non-commercial use, distribution, and reproduction in any medium, provided the original work is properly cited. 
Aspartic acid (Asp, D) is electrically similar to phosphorylated Ser (17). To construct a phosphomimetic form of PDH E1 subunit alpha 1 (PDHA1), we replaced the above described three Ser residues into Asp (S293D, S300D and S232D; hereafter, PDHA1 ${ }^{3 S D}$ ). A phosphomimetic PDHA1 is permanently similar to phosphorylated PDHA1 regardless of whether PDKs are active or not. Consequently, it could be resistant to PDK inhibitors. To ensure the function of PDHA $1^{3 \mathrm{SD}}$, endogenous PDHA1 was silenced by the CRISPR/Cas9 system. Subsequently, ectopic wildtype $\left(\mathrm{PDHA} 1^{\mathrm{WT}}\right.$ ) and $\mathrm{PDHA} 1^{3 \mathrm{SD}}$ were reconstructed. In this study, we aimed to discriminate the PDH activity-dependent cell death induced by established PDK inhibitors in human non-small cell lung cancer A549 cells.

\section{RESULTS}

\section{Silencing of endogenous PDHA1 in A549 cells}

To establish a PDH activity-dependent cell death screening system, the CRISPR/Cas9 system was applied to knock out the PDHA1 gene in human non-small cell lung cancer A549 cells. Four lentiCRISPR vectors containing PDHA1-targeting single guide RNAs (sgRNAs) were transfected into A549 cells (Supplementary Fig. 1). Next, the T7E1 assay was conducted to evaluate the site-specific cleavage. The substantial cleavage was detected in $\mathrm{A} 549 \mathrm{PDHA} 1^{\mathrm{KO}} \# 1$ cells (Fig. $1 \mathrm{~A}$ and Supplementary Fig. 2),
A)

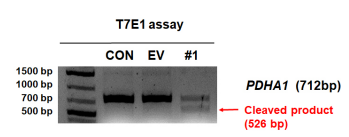

C)

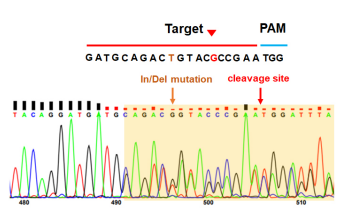

B)

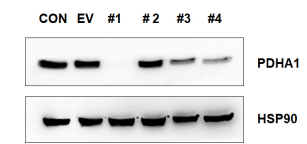

D)

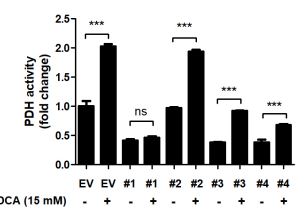

Fig. 1. CRISPR/Cas9-mediated PDHA1 knockout in A549 cells. PDHA1targeting lentiCRISPR vectors were transiently transfected into A549 cells as indicated. Untransfected and empty vector-transfected A549 cells (CON and EV, respectively) were used as a negative control. (A) Genomic DNA was extracted, and the target region of PDHA1 was amplified by polymerase chain reaction (PCR). PCR products were extracted and incubated with T7 Endonuclease I. The fragments are indicated by the red arrow. (B) Total PDHA1 expression was detected by western blot. HSP90 was used as an internal control. (C) Sequencing analysis was performed with genomic DNA derived from A549 PDHA $1^{\mathrm{KO}}$ (\#1) cells. The sgRNA-target sequence is marked with a red line; the PAM sequence is marked with a blue line; the yellow and red arrows indicate the in/del mutation and cleavage site in $\mathrm{A} 549 \mathrm{PDHA}^{\mathrm{KO}}(\# 1)$ cells, respectively. (D) $\mathrm{EV}$ and $\mathrm{PDHA} 1^{\mathrm{KO}}$ (\#1, \#2, \#3 and \#4) A549 cells were treated with or without DCA $(15 \mathrm{mM})$ for $24 \mathrm{~h}$. PDHA1 activity was determined using a commercially available PDH activity kit. The relative activities to DCA-free EV cells were calculated and are presented as means \pm SEM. $* * * \mathrm{P}<0.001$. but not in the \#2, \#3, or \#4 group (data not shown). In parallel with the T7E1 assay, PDHA1 protein expression completely disappeared in A549 PDHA $1^{\mathrm{KO}}$ \#1 cells (Fig. 1B). Sequence analysis also revealed that In/Del mutation occurred at the sgRNA-targeted site in A549 PDHA $1^{\mathrm{KO}} \# 1$ cells (Fig. 1C). To further ensure PDH knockout in transfected A549 cells, PDH activity was measured with or without DCA treatment. PDH activity was not restored by DCA in A549 PDHA $1^{\mathrm{KO}} \# 1$ cells indicating that the deletion of PDHA1 successfully blocks Acetyl-CoA synthesis (Fig. 1D). Thus, A549 PDHA1 ${ }^{\mathrm{KO}} \# 1$ cells were chosen for further study.

\section{The comparison of PDHA $1^{\text {WT }}$ and PDHA1 ${ }^{3 S D}$ A549 cells}

To establish the constitutively inactive PDHA1 vectors, three Ser residues - S232, S293 and S300 - were substituted with Asp to mimic their phosphorylations (18). WT and 3SD PDHA1 were re-introduced in $\mathrm{A} 549 \mathrm{PDHA}^{\mathrm{KO}} \# 1$ cells and named
A)

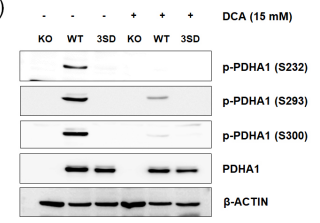

C)

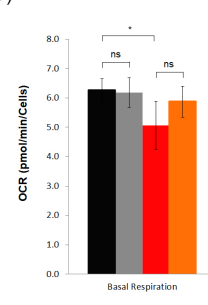

D)

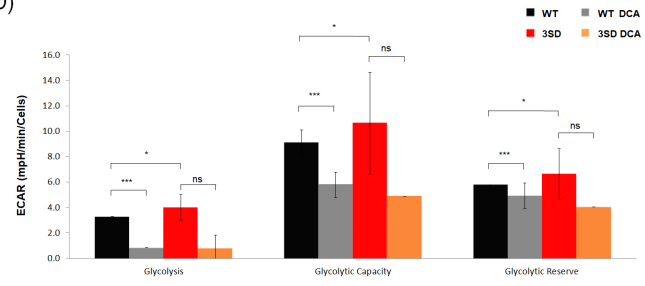

Fig. 2. Construction and analyses of phosphomimetic PDHA1-expressing A549 cells. PDHA $1^{\text {WT }}$ or phosphomimetic PDHA1 ${ }^{3 \mathrm{SD}}$ were reintroduced into PDHA $1^{\mathrm{KO}}$ A549 cells. (A, B) PDHA $1^{\mathrm{KO}}$, PDHA $1^{\text {WT }}$ and PDHA1 ${ }^{3 S D}$ A549 cells were treated with DCA (15 mM) for $24 \mathrm{~h}$ as indicated. (A) The levels of phosphorylated serines in PDHA1 (S232, S293 and S300), and total PDHA1 were examined by western blot analysis. $\beta$-ACTIN was used as an internal loading control. (B) $\mathrm{PDH}$ activity was measured by a commercially available PDH activity assay kit. The relative activities on DCA-free PDHA $1^{\mathrm{KO}}$ A549 cells were calculated and are shown as means \pm SEM. $* \mathrm{P}<0.05$ compared with the KO control ( $1^{\text {st }}$ line), ${ }^{\# \# \# ~} \mathrm{P}<0.001$ compared with the WT control $\left(2^{\text {nd }}\right.$ line). (C, D) OCR (C) and ECAR (D) in PDHA $1^{\text {WT }}$ and PDHA1 ${ }^{3 S D}$ A549 cells were measured with or without DCA $(15 \mathrm{mM})$ as indicated. Values are presented as means \pm SD. 


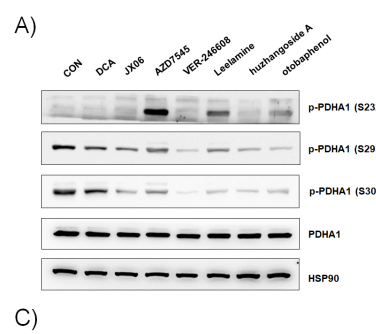

B)

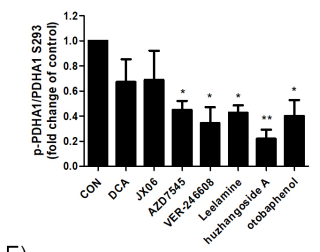

E)

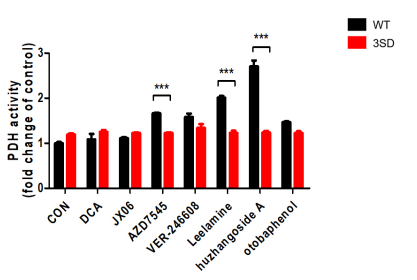

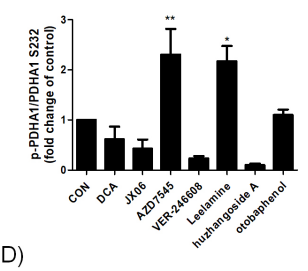

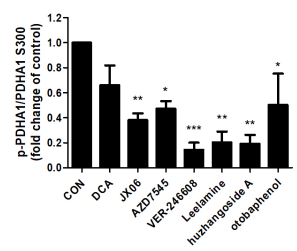

$\mathbf{m}_{3 s 0}^{\mathrm{wr}}$

Fig. 3. Specificity assessments for targeting $\mathrm{PDH}$ using PDHA1-manipulated A549 cells. (A-D) PDHA1 ${ }^{\text {WP }}$ A549 cells were treated with DCA $(10 \mu \mathrm{M})$, JX06 $(10 \mu \mathrm{M})$, AZD7545 $(10 \mu \mathrm{M})$, VER-246608 (10 $\mu \mathrm{M})$, Leelamine $(10 \mu \mathrm{M})$, huzhangoside $\mathrm{A}(1 \mu \mathrm{M})$ and otobaphenol $(10 \mu \mathrm{M})$ for $4 \mathrm{~h}$ as indicated. (A) The levels of phosphorylated serines in PDHA1 (S232, S293 and S300) and total PDHA1 were examined by western blot analysis. HSP90 was used as an internal control. (B-D) The densitometric analyses (p-PDHA1/PDHA1) from three independent experiments were performed and are presented by means \pm SEM. $* P<0.05, * * P<0.01 * * * P<0.001$. (E) $\mathrm{PDHA} 1^{\mathrm{WT}}$ and PDHA ${ }^{3 \mathrm{SD}}$ A549 cells were treated with DCA $(10 \mu \mathrm{M})$, JX06 $(10 \mu \mathrm{M})$, AZD7545 $(10 \mu \mathrm{M})$, VER-246608 $(10 \mu \mathrm{M})$, Leelamine $(10 \mu \mathrm{M})$, huzhangoside $\mathrm{A}(1 \mu \mathrm{M})$ and otobaphenol $(10 \mu \mathrm{M})$ for 4 $\mathrm{h}$ as indicated. PDH activity was measured by a commercially available PDH activity assay kit. The relative activities to untreated PDHA1 ${ }^{\mathrm{WT}}$ cells were calculated and are presented as means \pm SEM. $* * * P<$ 0.001 compared with the WT control.

$\mathrm{PDHA}^{\mathrm{WT}}$ and $\mathrm{PDHA}{ }^{3 \mathrm{SD}}$ cells, respectively. The phosphorylations of PDHA1 (S232, S293 and S300) were detected in PDHA $1^{\text {WT }}$ cells and phosphorylations of all Ser residues were suppressed by DCA (Fig. 2A). Following the phosphorylation, the PDH activity was increased by DCA in PDHA $1^{\mathrm{WT}}$ cells, but not in $\mathrm{PDHA} 1^{3 \mathrm{SD}}$ cells. However, the residual PDH activity of PDHA $1^{3 \mathrm{SD}}$ cells was still observed regardless of DCA treatment (Fig. 2B). Next, OXPHOS levels, based on the oxygen consumption rate (OCR), were evaluated (Supplementary Fig. 3A). The basal respiration was higher in PDHA $1^{\mathrm{WT}}$ cells than in PDHA $1^{3 \mathrm{SD}}$ cells, and DCA significantly increased respiratory capacity in PDHA $1^{\text {WT }}$ cells compared to PDHA ${ }^{3 S D}$ cells. Moreover, PDHA $1^{\text {WT }}$ cells produced more ATP than $\mathrm{PDHA}^{3 \mathrm{SD}}$ cells, and DCA significantly enhanced ATP production in PDHA $1^{\mathrm{WT}}$ cells (Fig. 2C). Glycolysis was further examined by measuring the extra-

A)
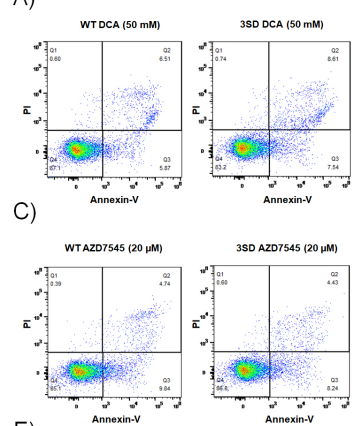

E)

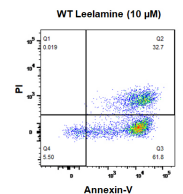

G)
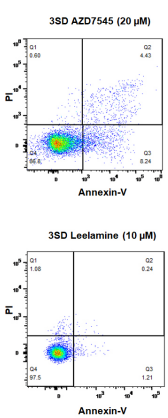

G)
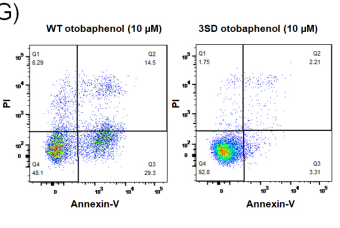

B)

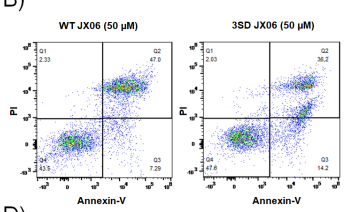

D)
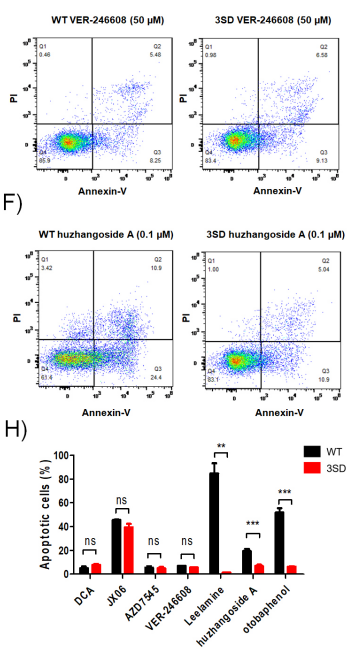

Fig. 4. Validations for PDH-dependent apoptotic cell death using PDHA1-manipulated A549 cells. PDHA $1^{\text {WT }}$ (WT) and PDHA1 ${ }^{\text {3SD }}$ (3SD) A549 cells were treated with DCA $(50 \mathrm{mM} ; \mathrm{A})$, JX06 $(50 \mu \mathrm{M} ; \mathrm{B})$, AZD7545 (20 $\mu \mathrm{M} ; \mathrm{C})$, VER-246608 (50 $\mu \mathrm{M} ; \mathrm{D})$, Leelamine (10 $\mu \mathrm{M} ; \mathrm{E})$, huzhangoside $\mathrm{A}(0.1 \mu \mathrm{M} ; \mathrm{F})$ and otobaphenol $(10 \mu \mathrm{M} ; \mathrm{G})$ for $24 \mathrm{~h}$. The cells stained with Annexin V and propidium iodide (PI) were analyzed by flow cytometry. $(\mathrm{H})$ The percent frequency of Annexin V-positive apoptotic cells is shown as means $\pm \mathrm{SEM}$. $* * \mathrm{P}<$ $0.01 ; * * * \mathrm{P}<0.001$

cellular acidification rate (ECAR) (Supplementary Fig. 3B). The basal glycolytic capacity level was higher in PDHA $1^{3 \mathrm{SD}}$ cells than in PDHA ${ }^{\text {WT }}$ cells, and DCA significantly reduced glycolytic capacity in PDHA1 ${ }^{\text {WT }}$ cells (Fig. 2D). These results indicate that ectopic PDHA $1^{\mathrm{WT}}$ and PDHA $1^{3 \mathrm{D} D}$ were successfully reintroduced into PDHA $1^{\mathrm{KO}}$ cells and that PDHA $1^{3 \mathrm{SD}}$ was less sensitive to DCA, a well-known PDK inhibitor.

\section{The activation of PDHA1 by different PDK inhibitors leads to different outcomes}

To analyze the correlation between PDHA1 phosphorylation status and activity alteration, the same concentration of PDK inhibitors of $10 \mu \mathrm{M}$ was selected, except for huzhangoside A for which $1 \mu \mathrm{M}$ was selected owing to its high cytotoxic sensitivity. AZD7545, VER-246608, Leelamine, huzhangoside A and otobaphenol markedly reduced the S293 and S300 phosphorylations of PDHA1 (Fig. 3A-D). Interestingly, S232 phosphorylation of PDHA1 was increased by AZD7545 and Leelamine. AZD7545, Leelamine, otobaphenol and huzhangoside A increased PDH activity in PDHA $1^{\text {WT }}$ cells that were inversely related to the S293 phosphorylation status of PDHA1 (Fig. 3E). 
However, dephosphorylations at S232 and S300 were not consistent with the enhancement of PDH activity (Fig. 3B, D, E). Additionally, PDH activity in PDHA ${ }^{3 \mathrm{SD}}$ cells was not altered by PDK inhibitors (Fig. 3E). To further investigate whether cell death could be accompanied with $\mathrm{PDH}$ activation, cell viabilities were compared between PDK inhibitors treated PDHA $1^{\text {WT }}$ and $\mathrm{PDHA}^{3 \mathrm{SD}}$ cells (Supplementary Fig. 4). Although the $50 \%$ cytotoxic concentration $\left(\mathrm{CC}_{50}\right)$ was higher in PDHA ${ }^{3 \mathrm{SD}}$ cells than in PDHA $1^{\text {WT }}$ cells, DCA, AZD7545 and VER-246608 were less toxic than other drugs, and JX06 showed similar $\mathrm{CC}_{50}$ in both PDHA $1^{\mathrm{WT}}$ and PDHA ${ }^{3 \mathrm{SD}}$ cells (Supplementary Table 3). Finally, apoptosis was measured by flow cytometry and the concentration of PDK inhibitors was determined as given in Supplementary Fig. 4. Consistent with the cell viability measurements, apoptosis was seldom induced by DCA, AZD7545 and VER-246608 (Fig. 4A-D). In contrast, Leelamine, huzhangoside A and otobaphenol-induced significantly higher apoptosis in $\mathrm{PDHA}^{\mathrm{WT}}$ cells than in PDHA $1^{3 \mathrm{SD}}$ cells (Fig. 4E-G). Thus, Leelamine, huzhangoside $A$ and otobaphenol induced PDH activity dependent apoptosis. However, AZD7545, VER-246608 and DCA successfully enhanced PDH activity but failed to induce apoptotic cell death (Fig. 4H). Based on our results, PDH activation by different PDK inhibitors could lead to different outcomes. In summary, PDHA $1^{\mathrm{WT}}$ and $\mathrm{PDHA} 1^{3 \mathrm{SD}}$ cells can be used as a cell-based validation system for cancer treatment, however PDK inhibitors and the regulation of $\mathrm{PDH}$ activity requires more in-depth research.

\section{DISCUSSION}

PDKs are potential targets for cancer and metabolic disease treatment (19). PDK1 functions as an oncogene that supports cancer cell proliferation and metastasis in non-small cell lung cancer (10). In our recent study, A549 cells were the most sensitive to ilimaquinone, one of our novel PDK inhibitors, among several cancer cell lines. Thus, we selected A549 cells to establish a cell-based screening system to validate $\mathrm{PDH}$ activity-dependent cell death. In addition, we aimed to discover new PDK inhibitors by evaluating our PDHA $1^{\mathrm{WT}}$ and $\mathrm{PDHA} 1^{3 \mathrm{SD}}$ cell-based screening system using well-known PDK inhibitors including DCA, JX06, AZD7545, VER-246608, Leelamine, huzhangoside $A$ and otobaphenol. Therefore, at first these drugs were merely used to evaluate the PDHA $1^{\mathrm{WT}}$ and $\mathrm{PDHA} 1^{3 \mathrm{SD}}$ cells, rather than to validate their efficacy.

The PDC is a complex of three components, $\mathrm{PDH}$ (E1), dihydrolipoyl acetyltransferase (E2) and dihydrolipoyl dehydrogenase (E3) (20). The PDC activity is strictly regulated by two enzymes, PDK and PDP, mainly through phosphorylation (inhibition) and dephosphorylation (activation) of three well-known Ser residues (S232, S293 and S300) in PDHA1 (21). Phosphorylation(s) of any of the three sites can suppress PDH activity (22). In this study, we replaced all three Ser sites with Asp, and PDHA $1^{3 S D}$ was not affected by PDKs (Fig. 2B and 3C). Phosphorylation is responsible for rapid and timely inhibition, thus long-term phosphorylation, such as mutations, could result in unknown metabolic changes. In this study, there was residual $\mathrm{PDH}$ activity in PDHA $1^{3 \mathrm{SD}}$ but not in PDHA $1^{\mathrm{KO}}$ (Fig. 2B). A very recent study has shown that AMPK can activate PDC by phosphorylating S295 and S314 in PDHA1 (23). The level of p-AMPK $\alpha$ was higher in PDHA ${ }^{3 S D}$ cells than in PDHA $1^{\text {WT }}$ cells (data not shown). To this end, our data might reveal the complex regulation of $\mathrm{PDH}$ activity, more than the known regulating mechanism(s) by PDKs through the well-known three Ser residues. We suspect that the regulation of $\mathrm{PDH}$ activity requires more in-depth research. A vigorous study using our PDHA $1^{3 \mathrm{SD}}$ cells could reveal the regulation of PDH activity by other pathway(s).

PDKs are engaged to the PDC by precise binding to the inner lipoamide domain of the $\mathrm{PDH}-\mathrm{E} 2 / \mathrm{E} 3$ binding protein (E3BP) core, and they effectively phosphorylate the PDHA1 (4). Elevated expressions of PDKs lead to a shift to glycolysis in cancer. PDK1 can phosphorylate all three phosphorylation sites, whereas PDK2, PDK3 and PDK4 can phosphorylate just two sites, S293 and S300 (24). The phosphorylation at S293 was faster than that at S300 and S232 (25), and the deactivating effect of phosphorylation at S300 or S232 was weaker than that at S293 (26). A previous study reported that reduction of phosphorylations at S232 and S300 did not affect PDH activity (27). Therefore, S293 is the major site for regulating PDH activity by phosphorylation (28). Consistent with many previous studies, our data indicate that the enhancement of PDH activity was most closely related to phosphorylation at S293 (Fig. 3). In this study, we found that phosphorylation of S232 was increased, rather than reduced, by AZD7545 and Leelamine through an unknown underlying mechanism(s) (Fig. $3 \mathrm{~B}, \mathrm{C})$. Thus far, the mechanism of PDH activity regulation by phosphorylation at S232 and/or S300 in PDHA1 remains unclear.

There are four binding sites in PDKs those are critical for its activity regulation, including the pyruvate-binding domain (N-terminal regulatory domain), lipoamide-binding domain, nucleotidebinding domain (C-terminal catalytic domain) and allosteric CoA-binding site (29). DCA (30) binds to pyruvate-binding pocket; AZD7545 (31) targets the lipoamide-binding pocket; while JX06 (32), VER-246608 (33) and huzhangoside A (14) target the ATP-binding pocket. Although DCA is the most investigated PDK1 inhibitor, its enzymatic inhibition of PDKs requires the millimolar level, whereas the enzymatic inhibitions of JX06, AZD7545 and VER-246608 to PDKs are enough for nanomolar scales (Supplementary Table 4). However, the anticancer efficacy of VER-246608 and AZD7545 seemed obscure, consistent with previous studies $(33,34)$, and JX06 was little specific to PDHA $1^{\text {WT }}$ cells than to PDHA $1^{3 \mathrm{SD}}$ cells. In another aspect, the in vitro inhibitory efficacy of huzhangoside A and otobaphenol on PDKs activity has not been explored. Meanwhile, AZD7545 and VER-2466608 sufficiently increased PDH activity without inducing severe cancer cell death. These results indicate that the correlation between $\mathrm{PDH}$ activation and activity-related cancer cell death by PDK inhibitors could be poor, 
and more studies are necessary to confirm such correlation.

This study is not intended to validate the effectiveness of drugs, such as DCA, JX06, AZD7545, VER-246608, Leelamine and otobaphenol, and has several other limitations. First, only one NSCLC cell line was used. A further extensive study using various types of human cancer cells could solidify our cellbased validation system for cancer treatable PDK inhibitors. Second, the link between PDH activation and its activity-related cancer cell death induced by PDK inhibitors is unclear. Third, another way of PDH activity regulation(s) (except for S232, S293 and S300) by investigated PDK inhibitors is still possible. This also requires further experiments. Clinical use of all current PDK inhibitors face the challenge of pharmacokinetics, potency, selectivity and efficacy (19). Despite these limitations, our study can contribute to new insights and strategies for developing of efficient and low-toxic PDK inhibitors. In conclusion, phosphomimetic PDHA $1^{3 \mathrm{SD}}$ reveals the complexity of its activity regulation, and PDHA $1^{\mathrm{WT}}$ and PDHA $1^{3 \mathrm{SD}}$ A549 cells can be used as a cell-based PDK inhibitor-evaluation system for cancer treatment and a valuable tool for PDHA1 biochemical research in cancer cell biology.

\section{MATERIALS AND METHODS}

\section{Cell culture}

A549 cells (Korean Cell Line Bank, Seoul, Korea; 10185) were cultured in RPMI 1640 medium (Welgene, Gyeongsan, Korea) with $10 \%$ heat-inactivated fetal bovine serum (FBS; Gibco, Thermo Fisher Scientific, Waltham, MA) and 1\% penicillin/streptomycin (Gibco). Platinum-A (Plat-A) (Cell Biolabs, San Diego, CA; RV102) cells were grown in Dulbecco's Modified Eagle's Medium (Welgene) supplemented with 10\% FBS (Gibco) and 1\% penicillin/streptomycin and selected by $5 \mu \mathrm{g} / \mathrm{ml}$ puromycin (SigmaAldrich) and $10 \mu \mathrm{g} / \mathrm{mL}$ blasticidin (Sigma-Aldrich) prior to produce retroviruses. All cells were incubated at $37^{\circ} \mathrm{C}$ in with $5 \% \mathrm{CO}_{2}$.

\section{Cellular energy metabolism by Seahorse XFe96 Analyzer}

The OCR and ECAR were measured using an XFe96 Extracellular Flux Analyzer (Seahorse Bioscience, Billerica, MA) according to the manufacturer's protocol. Briefly, cells were seeded in XFe96 culture plates at a density of $1.6 \times 10^{4}$ cells per well. The next day, the medium was replaced with XF base medium (pH 7.4, Seahorse Biosciences) supplemented with $25 \mathrm{mM}$ $D$-glucose (Sigma-Aldrich; G7528), $1 \mathrm{mM}$ sodium pyruvate (Sigma-Aldrich; S8636) and 1 X GlutaMAX (Gibco; 35050), or with XF Base Media ( $\mathrm{pH} 7.4$, Seahorse Biosciences) with 1 $\mathrm{mM}$ glutamine (Sigma-Aldrich; G8540) following the manufacturer's protocol. To measure the OCR and ECAR, the compounds and metabolites used in this study were as follows: DCA (15 mM, Sigma-Aldrich; 347795), oligomycin A ( $1 \mu \mathrm{M}$, Sigma-Aldrich; 75351), trifluoromethoxy carbonylcyanide phenylhydrazone (FCCP; $2 \mu \mathrm{M}$, Sigma-Aldrich; C2920), rotenone (1 $\mu \mathrm{M}$, SigmaAldrich; R8875) and antimycin A (1 $\mu \mathrm{M}$, Sigma-Aldrich; A8674); $D$-glucose (10 mM, Sigma-Aldrich; G7528), oligomycin A (2 $\mu \mathrm{M}$, Sigma-Aldrich; O75351) and 2-deoxy-D-glucose (2-DG; $50 \mathrm{mM}$, Sigma-Aldrich; D6134), for OCR and ECAR, respectively. For the normalization, DAPI stained cells were counted by the microscopy automatically (BioTek Lionheart FX, The Netherlands).

\section{Detection of apoptotic cells by flow cytometry}

$\mathrm{PDHA}^{\mathrm{WT}}$ and PDHA1 ${ }^{3 \mathrm{SD}}$ A549 cells were treated with various concentrations of drugs for $24 \mathrm{~h}$. Apoptotic cells were examined using an apoptosis detection kit (BD Biosciences, San Jose, CA), detected by an Attune Acoustic Focusing Cytometer (Invitrogen), and analyzed using Flowjo software.

\section{Statistical analysis}

The $\mathrm{CC}_{50}$ of the drugs was calculated by excel curve fitting. The other analyses were carried out by GraphPad Prism software (GraphPad Software, San Diego, CA). The difference between the mean values of each group was analyzed using Student's t-test, and multi comparisons among groups were analyzed using one-way analysis of variance with Dennett. The minimum level of statistical significance was set at a $\mathrm{P}<$ 0.05 for all the analyses. All the experiments were independently conducted thrice. Further details were shown in the supplementary material.

\section{ACKNOWLEDGEMENTS}

This work was supported by the National Research Foundation of Korea (NRF) grants funded by the Korean government (MIST; 2020R1C1C1003703 to Sung-Jin Bae, 2019R1A2C2003624 and 2021R1A4A1025662 to Ki-Tae Ha).

\section{AUTHOR CONTRIBUTION}

S.-J.B. and K.-T.H. conceived and supervised the project. L.J. performed the majority of experiments and collected the data. L.J. and M.C. performed the FACS analysis. B.-S.K. and J.H.H. cloned vectors used for genome editing and reconstructing PDHA $1^{\text {WT }}$ and PDHA $1^{\text {SSD }}$. S.P. and I.K.L examined OCR and ECAR. L.J. and S.-J.B. wrote the manuscript. D.R., J.H.K, I.K.L. and K.-T.H analyzed the data and revised the manuscript. All the authors reviewed the manuscript and agreed to the submission.

\section{CONFLICTS OF INTEREST}

The authors have no conflicting interests.

\section{REFERENCES}

1. Zheng J (2012) Energy metabolism of cancer: glycolysis versus oxidative phosphorylation. Oncol Lett 4, 1151-1157

2. Vander Heiden MG, Cantley LC and Thompson CB (2009) Understanding the Warburg effect: the metabolic require- 
ments of cell proliferation. Science 324, 1029-1033

3. Kroemer G and Pouyssegur J (2008) Tumor cell metabolism: Cancer's Achilles' heel. Cancer Cell 13, 472-482

4. Gatenby RA and Gillies RJ (2004) Why do cancers have high aerobic glycolysis? Nat Rev Cancer 4, 891-899

5. Pelicano H, Martin D, Xu R, and and Huang P (2006) Glycolysis inhibition for anticancer treatment. Oncogene 25,4633

6. Jeoung NH (2015) Pyruvate dehydrogenase kinases: therapeutic targets for diabetes and cancers. Diabetes Metab J 39,188

7. Golias T, Papandreou I, Sun R et al (2016) Hypoxic repression of pyruvate dehydrogenase activity is necessary for metabolic reprogramming and growth of model tumours. Sci Rep 6, 1-11

8. Kolobova E, Tuganova A, Boulatnikov I and Popov KM (2001) Regulation of pyruvate dehydrogenase activity through phosphorylation at multiple sites. Biochem J 358, 69-77

9. Schell JC, Olson KA, Jiang L et al (2014) A role for the mitochondrial pyruvate carrier as a repressor of the Warburg effect and colon cancer cell growth. Mol Cell $56,400-413$

10. Liu T and Yin H (2017) PDK1 promotes tumor cell proliferation and migration by enhancing the Warburg effect in non-small cell lung cancer. Oncol Rep 37, 193200

11. Stacpoole PW (2017) Therapeutic targeting of the pyruvate dehydrogenase complex/pyruvate dehydrogenase kinase (PDC/PDK) axis in cancer. JNCl: J Natl Cancer Inst 109, 11

12. Sun W, Xie Z, Liu Y et al (2015) JX06 selectively inhibits pyruvate dehydrogenase kinase PDK1 by a covalent cysteine modification. Cancer Res 75, 4923-4936

13. Jeoung NH and Harris RA (2010) Role of pyruvate dehydrogenase kinase 4 in regulation of blood glucose levels. Diabetes Metab J 34, 274

14. Kwak $\mathrm{CH}$, Lee JH, Kim EY et al (2019) Huzhangoside A suppresses tumor growth through inhibition of pyruvate dehydrogenase kinase activity. Cancers 11, 712

15. Kwak $\mathrm{CH}$, Jin $\mathrm{L}$, Han JH et al (2020) Ilimaquinone induces the apoptotic cell death of cancer cells by reducing pyruvate dehydrogenase kinase 1 activity. Int J Mol Sci 21, 6021

16. Jin L, Kim EY, Chung T-W et al (2020) Hemistepsin A suppresses colorectal cancer growth through inhibiting pyruvate dehydrogenase kinase activity. Sci Rep 10, 1-12

17. Guerra-Castellano A, Díaz-Moreno I, Velázquez-Campoy A, Miguel A and Díaz-Quintana A (2016) Structural and functional characterization of phosphomimetic mutants of cytochrome $\mathrm{C}$ at threonine 28 and serine 47 . Biochim Biophys Acta-Bioenerg 1857, 387-395

18. Hitosugi T, Fan J, Chung TW et al (2011) Tyrosine phosphorylation of mitochondrial pyruvate dehydrogenase kinase 1 is important for cancer metabolism. Mol Cell 44, 864877

19. Wang X, Shen X, Yan Y and Li H (2021) Pyruvate dehydrogenase kinases (PDKs): an overview toward clinical applications. Biosci Rep 41, BSR20204402
20. McFate T, Mohyeldin A, Lu H et al (2008) Pyruvate dehydrogenase complex activity controls metabolic and malignant phenotype in cancer cells. J Biol Chem 283, 22700-22708

21. Zimmer AD, Walbrecq G, Kozar I, Behrmann I and Haan C (2016) Phosphorylation of the pyruvate dehydrogenase complex precedes HIF-1-mediated effects and pyruvate dehydrogenase kinase 1 upregulation during the first hours of hypoxic treatment in hepatocellular carcinoma cells. Hypoxia 4, 135

22. SALE GJ and Randle PJ (1981) Analysis of site occupancies in [32P] phosphorylated pyruvate dehydrogenase complexes by aspartyl-prolyl cleavage of tryptic phosphopeptides. Eur J Biochem 120, 535-540

23. Cai Z, Li CF, Han F et al (2020) Phosphorylation of PDHA by AMPK drives TCA cycle to promote cancer metastasis. Mol Cell 80, 263-278. e267

24. Sradhanjali S and Reddy MM (2018) Inhibition of pyruvate dehydrogenase kinase as a therapeutic strategy against cancer. Curr Top Med Chem 18, 444-453

25. Korotchkina LG and Patel MS (1995) Mutagenesis studies of the phosphorylation sites of recombinant human pyruvate dehydrogenase. Site-specific regulation. J Biol Chem 270, 14297-14304

26. Korotchkina LG and Patel MS (2001) Site specificity of four pyruvate dehydrogenase kinase isoenzymes toward the three phosphorylation sites of human pyruvate dehydrogenase. J Biol Chem 276, 37223-37229

27. Jing E, O'Neill BT, Rardin MJ et al (2013) Sirt3 regulates metabolic flexibility of skeletal muscle through reversible enzymatic deacetylation. Diabetes 62, 3404-3417

28. Kato M, Wynn RM, Chuang JL et al (2008) Structural basis for inactivation of the human pyruvate dehydrogenase complex by phosphorylation: role of disordered phosphorylation loops. Structure 16, 1849-1859

29. Jeoung $\mathrm{NH}$ (2015) Pyruvate dehydrogenase kinases: therapeutic targets for diabetes and cancers. Diabetes Metab J 39, 188-197

30. Li J, Kato M and Chuang DT (2009) Pivotal role of the C-terminal DW-motif in mediating inhibition of pyruvate dehydrogenase kinase 2 by dichloroacetate. J Biol Chem 284, 34458-34467

31. Kato M, Li J, Chuang JL and Chuang DT (2007) Distinct structural mechanisms for inhibition of pyruvate dehydrogenase kinase isoforms by AZD7545, dichloroacetate, and radicicol. Structure 15, 992-1004

32. Sun W, Xie Z, Liu Y et al (2015) JX06 selectively inhibits pyruvate dehydrogenase kinase PDK1 by a covalent cysteine modification. Cancer Res 75, 4923-4936

33. Moore JD, Staniszewska A, Shaw T et al (2014) VER-246608, a novel pan-isoform ATP competitive inhibitor of pyruvate dehydrogenase kinase, disrupts Warburg metabolism and induces context-dependent cytostasis in cancer cells. Oncotarget 5, 12862

34. Mayers R, Butlin R, Kilgour E et al (2003) AZD7545, a novel inhibitor of pyruvate dehydrogenase kinase 2 (PDHK2), activates pyruvate dehydrogenase in vivo and improves blood glucose control in obese (fa/fa) Zucker rats. Biochem Soc Trans 31, 1165-1167 